\title{
Linear Regression Line based Partial Face Recognition
}

\author{
Naveena M. \\ Department of Studies in \\ Computer Science, \\ Manasagagothri, \\ Mysore.
}

\author{
G. Hemantha Kumar \\ Department of Studies in \\ Computer Science, \\ Manasagagothri, \\ Mysore.
}

\author{
P. Nagabhushan \\ Department of Studies in \\ Computer Science, \\ Manasagagothri, \\ Mysore.
}

\begin{abstract}
Various techniques have been proposed in the literature to detect and recognize full face images. However, only few attempts reported towards the problem of identifying and recognizing partial and occluded face images. In this direction, in this Paper, propose a method for recognition of a face under partial visibility. Initially, for every full face image in the training set, its intensity image is processed to obtain a histogram which represents relative frequency of occurrence of various gray levels in it. A cumulative histogram is generated by using the intensity histogram. Further, slope and intercept values are computed using a regression line fitted on the cumulative histogram. For every person, the slope and intercept values obtained for different training samples are aggregated to form an interval valued feature vector which is stored as the representative in the knowledgebase. During testing, slope and intercept values of a given partial face image are compared against the stored intervals of the training samples to recognize the person. The proposed algorithm has been experimentally validated on AR face dataset and results obtained are highly satisfactory.
\end{abstract}

\section{Keywords}

Partial face recognition, Linear regression, Slope, Intercept, interval valued features.

\section{INTRODUCTION}

Trustworthy authentication of a person has been a motivating goal and is becoming more important because of its immense need in defense. Defense is a facet that is given top priority by organizations, learning institutions, political and administration in personal identity and forgery issues. Face recognition is the task of identifying an already recognized face as a known or unknown face and it is an easy task for humans. But most difficult and challengeable task is to identify a face in occluded conditions with respect to Pose, Illumination, Brightness, Light scatter, Interferences etc. To overcome these types of problems, we need an accurate face recognition system which recognizes the face even with partial information. Some illustrations are shown in Figure 1.

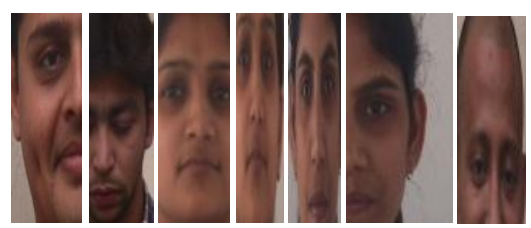

Fig 1: Partial faces of seven persons representing seven different cases

To build face identification and recognition pertinent to the real-life scenario, several works have been proposed to test partial face images. Lin and Ming [1] utilizes the extension of
Posterior Union Model (PUM) and similarity measure is resolute amongst the class containing single sample [2] per class. This paper has tried to overcome the problems of single training images and use of large feature vectors. Also, this method does not assume before where the partial occlusion would exist. Experiments performed on XM2VTS dataset and AR face database obtained recognition rates of $98.8 \%$ and 91.5\%, respectively. In [3], Marsico et al. explores Partitioned Iterated Function System (PIFS) based face recognition. PIFS based algorithms work out self-similarities among the representation and establish a relationship between the squares of grid region. Individual components of face such as eyes, mouth and nose are handled locally as PIFS algorithm is sensitive to occlusions. Further, distortions are removed by unplanned distance measure. To deal with face occlusions, various methodologies are utilized such as Sparse Representation based Classification (SRC) for face reconstruction $[4,5,6,7,8]$. The prime work was to design sparse representation into face recognition area, [9] where sparse representation was utilized to renovate and rebuild occluded or blemished facial images as well as to align testing face images to gallery images. These approaches would progress the recognition performance in case of partially occluded images and due to some incontinence to see and recognize the part of the image such as crowd, video surveillance, different facial expressions yet they are designed for near-frontal face recognition and they would fail if the probe image is an random face scrap. In contrast to these methods, our method recognizes the given partial face image directly without manual alignment using our robust feature set.

Since many years, several research have been carried out in developing systems to facilitate the automatic handling of great volume of full face images by means of no bad illumination, poses, intensity values of the image. Martinez and Quintilian [10] have proposed a methodology using local approaches to recognize partially occluded facial images. The main difference between our method and other local approaches is that, we use local linear regression concept to solve the partial faces recognition difficulties.

The variations and differentiation in manifestation caused by pose are closely related to the 3-D face modeling, it is a usual idea to get better 3-D model from the input 2-D face image. Thus, implicit views under any viewpoint are easily generated by using graphic rendering techniques [11], [12], [13]. But in our proposed system we describe the mathematical features such as slope and intercepts range values using parameter estimation. In this work, we first try to devise the relationship between a non frontal face image with a specific pose, part of the image illumination and its frontal equivalent based on the image structure model. We considered the existence of a linear mapping between the non frontal face images as 
training and the frontal one for a specific face under the same illumination is referred to as testing or sampling. Though the linear operators for a specific pose are varying for discrete personnel, they are quite similar due to the normal similarity of all the 3-D face shapes. Thus, a regression-based solution is intuitively applicable to estimate, for a novel person, the linear operator, which can be used for virtual view generation and this model mainly depending on to get a good result. Linear regression models are fitted using the least squares approach, but they may also be fitted in other ways, such as by minimizing the lack of fit in some other least absolute deviation regression or by minimizing a penalize description of the least squares as in ridge regression. Furthermore, the method is inspired by the idea that the linear mapping between non frontal patches and frontal patches maintains better than that of the global case in the case of coarse alignment. Compared with other approaches [14,15], our method is more efficient since only simple linear regression is needed. In addition, it is much easier to implement, considering that it requires only the centers of the two eyes for alignment rather than accurate face alignment, as is mandatory for LOC method.

Face detection is normally formulated as a classification.

\section{PROPOSED MODEL}

For our purpose, face recognition is actually an occluded or partial face image problem. Generally, there will be eight different cases of partial or occluded face images as follows. Top left side, Top right side, Bottom left side, Bottom right side ,Both Top left and right side, Both Bottom left and right side, Top left and Bottom left side, Top right and Bottom right side. Hence, all the cases need to be taken into account for efficient face recognition

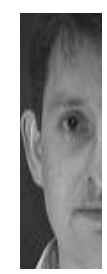

(a)

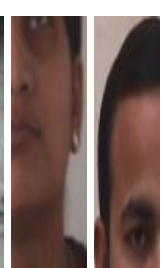

(b)

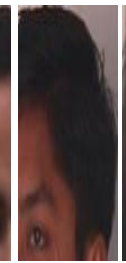

(d)

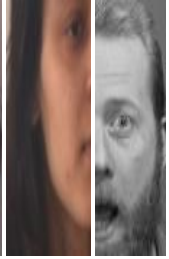

(e) (f)

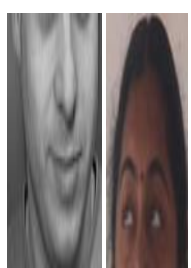

(g)
Fig.2 (a)-(h) shows Parameter extraction: Represents the eight cases of partial faces. And

Shows linear regression best line fitting: Comparison of two values usually $x$ and $y$, and the consistent change between those values. This full and partial face images shows the feature extraction: parameter matching such as slope and intercepts.

\subsection{Data Sets}

AR dataset: The AR dataset contains 80 subjects, including 70 male and 10 female, respectively. For each subject, there are 10 face pictures taken in to eight different sessions (each session has partial face images). Out of eight sections, there are 30 images with different occlusion conditions, some of the images have different facial expressions and disguise along with partial visibility such as illumination, poses and light scatter etc.

To trace human faces, facial features, such as eyes, nose and mouth, are usual candidate. But this facial appearance may change from time to time. Occlusion and non-rigidity are basic problems with these features. For example, when a person rotates his head, depth changes can wrap or occlude facial features. In this proposed model, facial image as divided in to eight partially occluded cases illustrated by Figure 2(a). In Figure 2(a), the top left side of a subject's face is shown; Figure 2(b) shows a top right frontal view of the face Figure 2(c) shows bottom left side 2(d) Right bottom side 2(e) shows both top left and right side in a face image 2(f) Both bottom left and right in a face image $2(\mathrm{~g})$ shows Top left and Bottom left in a facial image 2(h) shows Top right and Bottom right in a face image. Here, proposed system works for different partially visible cases.

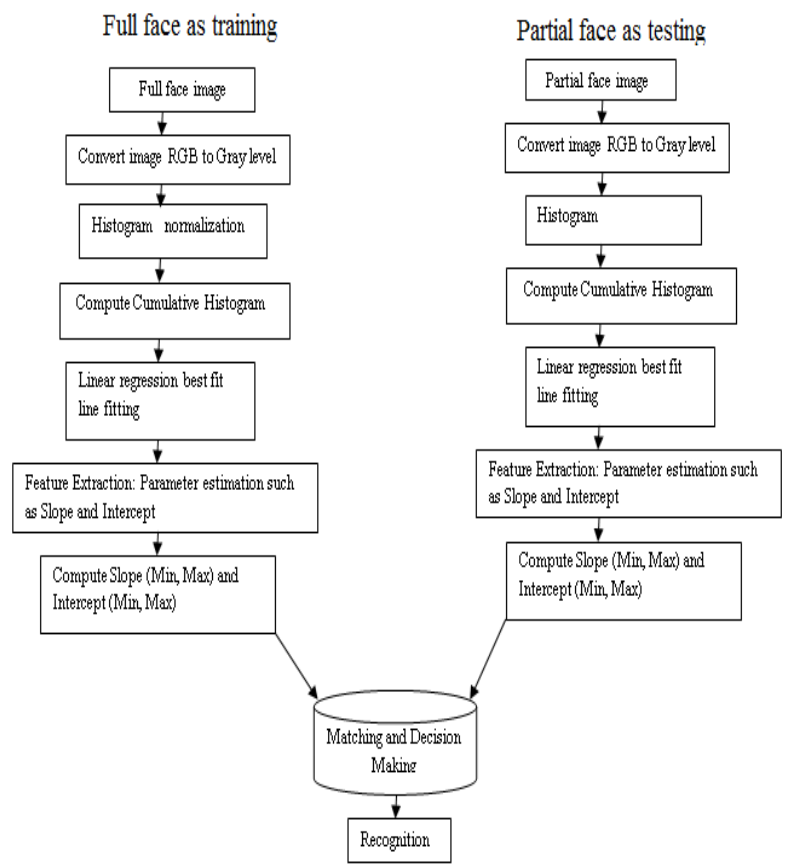

Fig 3. Architecture of Proposed Model

Few design approaches have been introduced to recognize partial face recognition, but in our model, we achieved face recognition under partial visibility like occluded image, part of the facial image and some facial expressions. Firstly we have taken full face as training set, part of the image as query set (for both we use AR database consisting 600 face images) then convert the colored images in to gray level images and normalize the histogram of an image it represents the relative frequency of occurrence of the various gray levels in the image. Subsequently, we have calculated cumulative histogram of the face images and we got features such as slope and intercept range (minimum and maximum) values which is done by fitting linear regression line for each image. Lastly, matching the features using decision making and compare range values of features. The proposed algorithm has been testing with AR dataset and results obtained are satisfactory compare to existing methodologies.

\subsection{Feature Extraction}

\subsubsection{Generating cumulative histogram}

In this section, discussing each input full-face image feature extraction. Initially, for every full face image in the training set, its intensity image is processed to obtain a histogram which represents relative frequency of occurrence of various gray levels in it. 

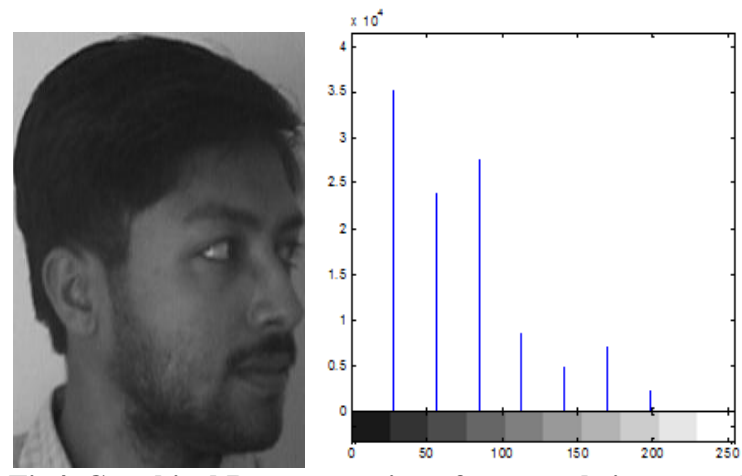

Fig4. Graphical Representation of gray scale image and histogram normalization

\subsubsection{Fitting Linear best fit Regression Line}

A statistical measure that attempts to determine the strength of the relationship between one dependent variable (usually denoted by $\mathrm{Y}$ ) and a series of other changing variables (known as independent variables). The two basic types of regression are linear regression and multiple regressions. Linear regression uses one independent variable to explain and/or predict the outcome of $\mathrm{Y}$, while multiple regressions uses two or more independent variables to predict the outcome. The general form of each type of regression is:

Linear Regression:

$$
\mathrm{Y}=\mathrm{a}+\mathrm{bX}+\mathrm{u}
$$

Multiple Regressions:

$$
\mathrm{Y}=\mathrm{a}+\mathrm{b}_{1} \mathrm{X}_{1}{ }^{+} \mathrm{b}_{2} \mathrm{X}_{2}+\mathrm{B}_{3} \mathrm{X}_{3}+\ldots+\mathrm{B}_{\mathrm{t}} \mathrm{X}_{\mathrm{t}}+\mathrm{u}
$$

In this model, we have to extract the features such as slope and intercept using linear regression best fit line
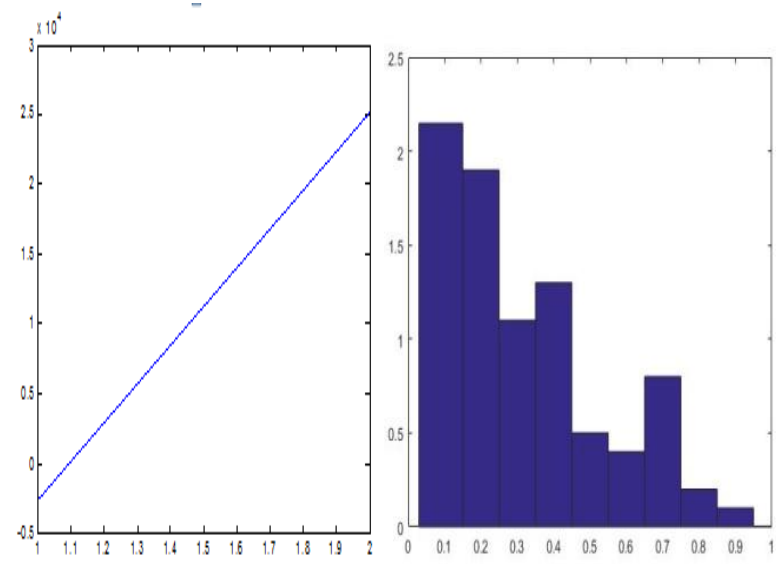

Fig5. Graphical Representation of Fitting regression on cumulative histogram.

\subsubsection{Slope and Intercept Computation}

Every straight line can be determined by an equation: $\mathrm{y}=\mathrm{mx}$ $+b$. The coordinates of each point on the line will solve the equation if you replacement them in the equation for $\mathrm{x}$ and $\mathrm{y}$.

The slope $m$ of this line - its steepness or slant - can be calculated like this:

\section{$m=\underline{\text { change in } y \text {-value }}$}

change in $x$-value
The equation of any straight line, called a linear equation, can be written as: $y=m x+b$, where $m$ is the slope of the line and $\mathrm{b}$ is the $\mathrm{y}$-intercept.

\subsubsection{Interval valued feature vector for representation}

Person $\mathrm{P}$ with $\mathrm{n}$ samples we create an interval $\mathrm{I}_{\mathrm{p}}=\left\{\left[\mathrm{S}_{\text {min, }}^{\mathrm{p}} \mathrm{S}^{\mathrm{p}}{ }_{\text {max }}\right]\right\}\left\{\left[\mathrm{I}_{\text {min }}^{\mathrm{p}}, \mathrm{I}_{\text {max }}^{\mathrm{P}}\right]\right\}$

Where $S_{\text {min }}^{\mathrm{p}}=\operatorname{Min}\left\{\mathrm{S}_{1}^{\mathrm{p}}, \mathrm{S}^{\mathrm{p}}{ }_{2, \ldots} \mathrm{S}_{\mathrm{n}}^{\mathrm{p}}\right\}$ and $\mathrm{S}_{\max }^{\mathrm{p}}=\operatorname{Max}\left\{\mathrm{S}_{1}^{\mathrm{p}}\right.$, $\left.\mathrm{S}_{2, \ldots .}^{\mathrm{p}} \mathrm{S}_{\mathrm{n}}^{\mathrm{p}}\right\}$

$\mathrm{I}^{\mathrm{p}}{ }_{\min }=\operatorname{Min}\left\{\mathrm{I}^{\mathrm{p}}{ }_{1}, \mathrm{I}^{\mathrm{p}}{ }_{2 \ldots \ldots} \mathrm{I}^{\mathrm{p}}{ }_{\mathrm{n}}\right\}$ and $\mathrm{I}^{\mathrm{p}}{ }_{\max }=\operatorname{Max}\left\{\mathrm{I}^{\mathrm{p}}{ }_{1}, \mathrm{I}^{\mathrm{p}}{ }_{2 \ldots \ldots}\right.$ $\left.\mathrm{I}_{\mathrm{n}}^{\mathrm{P}}\right\}$

\subsubsection{Testing}

Given a test face fq, test image has some features and similar process is applied to generate slope and intercept values $\mathrm{Sq}$ is matched with $\{[$ Spmin, Spmax $]\}$ and Iq is matched along with $\{[$ Ipmin, Ipmax $]\}$ it has to be matched against stored intervals of slope of different persons similarly, interval values of intercept for different persons are stored. We check whether the value of $\mathrm{Sq}$ is within the interval [Smin, Smax] using the following formula.

If $\mathrm{Smin}<\mathrm{Sq}<\mathrm{Smax}$, then it is considered as the test samples belongs to corresponding training face.

For Imin $<$ Iq $<$ Imax, if it is true for both $\mathrm{Sq}$, for face then the person corresponds face is recognized as that person.

\section{EXPERIMENTAL RESULTS}

The experimental results are reported in terms of correctly searched faces over the number of all faces in the available datasets. A total of $600 \mathrm{AR}$ face images were validated by creating the dataset contains 80 subjects, including 70 male and 10 female, respectively. Each person has 8 different partial occlusion cases .Out of 600 partial 1 face images $60 \%$ for training process. And these 6 training images correspond to the 8 different occluded and illuminations images. All of the images in the training set were frontal, with neutral expression and with occlusions. For the test stage, the remaining $40 \%$ face images were used for testing the algorithm. Figure 2(B) shows the Regression Line dataset with 80 samples and 2 Regression features such as slope and intercepts. The variance regression line is obtained by computing the variance for $\mathrm{y} 1$ and $\mathrm{y} 2$ if $\mathrm{x} 1$ and $\mathrm{x} 2$ are fixed or by computing the variance of $\mathrm{x} 1$ and $\mathrm{x} 2$ if $\mathrm{y} 1$, and $\mathrm{y} 2$ are fixed. In the experimentation we have fixed $\mathrm{y} 1$ and $\mathrm{y} 2$ to 0 and 1 respectively. Then the corresponding Regression line was considered for slope and variance computation. In figure $2 \mathrm{~A}$ shows the different partial visibility of faces.

In this proposed methodology, a single image is classified into 8 different partial cases that are shows in figure 2(A). (a), the top left side; Figure 2(b), shows a top right; 2(c) shows bottom left side; 2(d), Bottom right side; 2(e), shows both top left and right side; 2(f), Both bottom left and right; 2(g), shows Top left and Bottom left; 2(h), shows Top right and Bottom right sides in a single face image and we got recognition rate $68.25,67.50,69.25,70.50,77.50,77.75,83.25$ and 85.00 respectively, by using precision, recall and Fmeasure. 
Table 1.Comparing the Ground truth values with results

\begin{tabular}{|c|c|c|}
\hline Person & $\begin{array}{l}\text { Ground } \\
\text { Truth } \\
\text { Values }\end{array}$ & $\begin{array}{l}\text { Obtained } \\
\text { Results }\end{array}$ \\
\hline $\mathrm{p} 1$ & 1 & 2 \\
\hline $\mathrm{p} 2$ & 1 & 1 \\
\hline $\mathrm{p} 3$ & 1 & 1 \\
\hline $\mathrm{p} 4$ & 1 & 2 \\
\hline $\mathrm{p} 5$ & 1 & 2 \\
\hline $\mathrm{p} 6$ & 2 & 1 \\
\hline $\mathrm{p} 7$ & 2 & 2 \\
\hline $\mathrm{p} 8$ & 2 & 2 \\
\hline $\mathrm{p} 9$ & 2 & 2 \\
\hline $\mathrm{p} 10$ & 2 & 2 \\
\hline
\end{tabular}

Here ground truth values are nothing but the actual labels of the test face images. It has to be corresponds as person 1 it has person2.

The mathematical formulation is as follows

$$
\begin{gathered}
\text { Precision }=\frac{\mid\{\text { relevant face }\} \cap\{\text { retrieved face }\} \mid}{\mid\{\text { retrieved face }\} \mid} \\
\text { Recall }=\frac{\mid\{\text { relevant face }\} \cap\{\text { retrieved face }\} \mid}{\mid\{\text { relevant face }\} \mid} \\
F=\frac{2 * \text { Precision } * \text { Recall }}{\text { Precision+Recall }}
\end{gathered}
$$

Following abbreviations are used for recognition shows in table 2 .

Table2. Shows the Recognition Rate of different partial conditions

(Experiments' on AR database)

\begin{tabular}{|c|c|c|}
\hline $\begin{array}{c}\text { Sl. } \\
\text { No. }\end{array}$ & Partial Face Cases & $\begin{array}{c}\text { Recognition } \\
\text { Rate in \% }\end{array}$ \\
\hline 1 & Top left side & 68.25 \\
\hline 2 & Top right side & 67.50 \\
\hline 3 & Bottom left side & 69.25 \\
\hline 4 & Bottom right side & 70.50 \\
\hline 5 & $\begin{array}{c}\text { Both Top left and right } \\
\text { side }\end{array}$ & 77.50 \\
\hline 6 & $\begin{array}{c}\text { Both Bottom left and } \\
\text { right side }\end{array}$ & 83.25 \\
\hline 7 & $\begin{array}{c}\text { Top left and Bottom left } \\
\text { side }\end{array}$ & 85.00 \\
\hline 8 & $\begin{array}{c}\text { Top right and Bottom } \\
\text { right side }\end{array}$ & \multicolumn{2}{|c|}{} \\
\hline
\end{tabular}

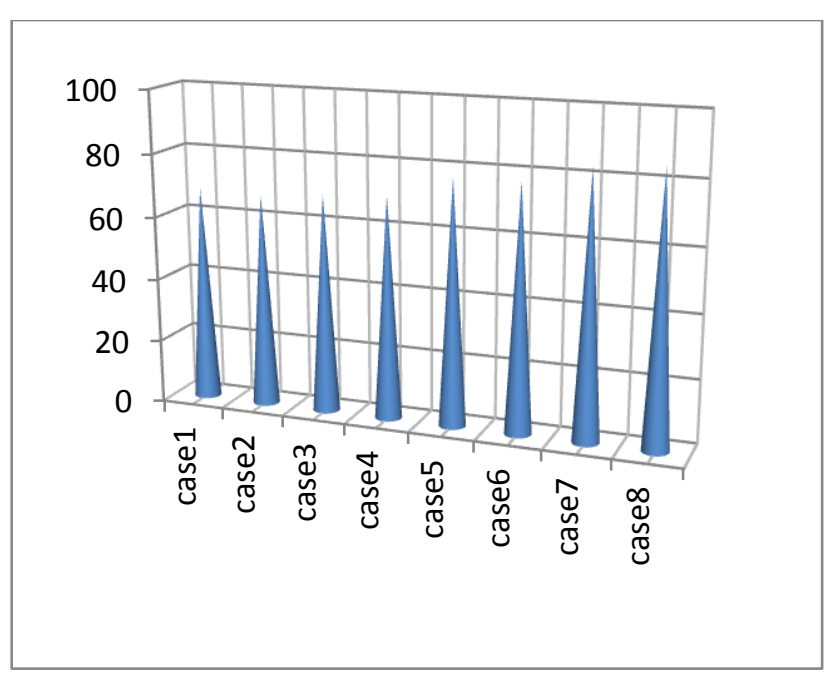

Fig.3. Evaluation of the recognition rate of Different partial conditions towards localization errors

\section{CONCLUSION}

Biometrics is used in the process of authentication of a person by verifying or identifying that a user requesting a network resource is who he or she claims to be and vice versa. In this proposed method, we implemented the partial face recognition system using Linear Regression Line approach. We have presented an approach for face recognition under partial visibility. This approach initially takes the color image and then converts it into gray level images which are subsequently processed to generate slope and intercept values as features. Experimental results show that the method has achieved the high accuracy specifically for Top left and Bottom left and Top right and Bottom right sides in a single face image.

The system successfully recognized the human faces in partially occluded conditions and worked better in different illuminations of face orientations.

\section{REFERENCES}

[1] Lin J., Ming J., and Crookes D., "Robust Face Recognition with Partially Occluded Images Based on A Single or a Small Number of Training Samples," in Proceedings of IEEE International Conference on Acoustics, Speech and Signal Processing, Taipei, pp. 881-884, 2009.

[2] H. Chen, P. Belhumeur, and D. Jacobs, an Search of Illumination Invariants, ${ }^{\circ}$ Proc. IEEE Conf. Computer Vision and Pattern Recognition,pp. 254-261, 2000.

[3] Acoustics, Speech and Signal Processing, Taipei, pp. 881-884, 2009.Marsico M., Nappi M., and Riccio D., "FARO: Face Recognition against Occlusions and Expression Variations," IEEE Transactions on System, Man, and Cybernetics-Part A: Systems and Humans, vol. 40, no. 1, pp. 121-132, 2010.

[4] P. Quintilian, A. N. Santa-Rosa, R. Guadagnin"Face recognition based on Eigen features", Proc.SPIE Vol. 4550 , p. 140-145, Image Extraction, Segmentation, and Recognition, 2001

[5] J. Wright, A. Y. Yang, A. Garnish, S. S. Sastry, and Y. Ma. Robust face recognition via sparse representation. PAMI, 31(2):210-227, 2009. 1, 6, 8

[6] L. Zhang, M. Yang, and X. Fang. Sparse representation or collaborative representation: Which helps face 
recognition? In ICCV, pages 471-478, 2011. 1, 6, 8

[7] E. Elhamifar and R. Vidal. Robust classification using structured sparse representation. In CVPR, pages 1873179, 2011.

[8] Y. Liu, F. Wu, Z. Zhang, Y. Zhang, and S. Yan. Sparse representation using nonnegative curds and whey. In CVPR, pages 3578-3585,2010. 1, 8

[9] K. Jiao, T.-H. Chan, and Y. Ma. Robust and practical face recognition via structured sparsely. In ECCV, pages 331-344. 2012. 1, 8

[10] J. Wright, A. Y. Yang, A. Ganesh, S. S. Sastry, and Y. Ma. Robust face recognition via sparse representation. PAMI, 31(2):210-227,2009.

[11] Aisha Azeem, Muhammad Sharif, A Survey: Face Recognition Techniques under Partial Occlusion The International Arab Journal of Information Technology, Vol. 11, No. 1, January 2014.
[12] G. B. Huang, M. Ramesh, T. Berg, and E. LearnedMiller. Labeled faces in the wild: A database for studying face recognition in unconstrained environments. Technical Report 07-49, University of Massachusetts, Amherst, 2007. 1, 6

[13] D. P. Huttenlocher, G. A. Klanderman, and W. J. Rockledge. Comparing images using the hausdorff distance. PAMI, 15(9):850-863,1993. 7

[14] K. Jia, T.-H. Chan, and Y. Ma. Robust and practical face recognition via structured sparsely. In ECCV, pages 331-344. 2012. 1,8

[15] L. Juan and O. Gwen. A comparison of sift, pca-sift and surf. International Journal of Image Processing (IJIP), 3(4):143-152, 2009.2 